\title{
Quantum fluctuations at the Planck scale
}

\author{
Fulvio Melia ${ }^{1,2, a_{\text {iD }}}$ \\ ${ }^{1}$ Department of Physics, the Applied Math Program, The University of Arizona, Tucson, AZ 85721, USA \\ ${ }^{2}$ Department of Astronomy, The University of Arizona, Tucson, AZ 85721, USA
}

Received: 18 February 2019 / Accepted: 17 May 2019 / Published online: 29 May 2019

(C) The Author(s) 2019

\begin{abstract}
The recently measured cutoff, $k_{\min }=4.34 \pm$ $0.50 / r_{\mathrm{cmb}}$ (with $r_{\mathrm{cmb}}$ the comoving distance to the last scattering surface), in the fluctuation spectrum of the cosmic microwave background, appears to disfavor slow-roll inflation and the associated transition of modes across the horizon. We show in this Letter that $k_{\min }$ instead corresponds to the first mode emerging out of the Planck domain into the semi-classical universe. The required scalar-field potential is exponential, though not inflationary, and satisfies the zero active mass condition, $\rho_{\phi}+3 p_{\phi}=0$. Quite revealingly, the observed amplitude of the temperature anisotropies requires the quantum fluctuations in $\phi$ to have classicalized at $\sim 3.5 \times 10^{15} \mathrm{GeV}$, consistent with the energy scale in grand unified theories. Such scalar-field potentials are often associated with Kaluza-Klein cosmologies, string theory and even supergravity.
\end{abstract}

\section{Introduction}

Three major satellite missions have now confirmed the lack of angular correlation at $\theta \gtrsim 60^{\circ}$ in the cosmic microwave background (CMB) [1-3], contrasting with the predictions of standard inflationary cosmology [4-7]. Though many possible instrumental and observational selection effects have been considered as the cause of this deficiency, today we appear to be left solely with cosmic variance as an explanation for the missing correlations. But even this conclusion disfavors the conventional picture at $\gtrsim 3 \sigma$. A recent indepth analysis of the Planck measurements [8] reveals that a far more likely reason for the missing angular correlations is a non-zero minimum wavenumber, $k_{\min }$, in the fluctuation power spectrum $P(k)$. This evidence now shows quite compellingly that a zero $k_{\min }$ is ruled out at a level of con-

Communicated by John Woodruff Simpson Fellow.

a e-mail: fmelia@email.arizona.edu

URL: http://www.physics.arizona.edu/ melia fidence exceeding $8 \sigma$. The Planck data suggest, instead, a cutoff $k_{\min }=4.34 \pm 0.50 / r_{\mathrm{cmb}}$, where $r_{\mathrm{cmb}}$ is the comoving distance to the surface of last scattering.

This measurement disfavors basic slow-roll inflation because, in the standard picture, $k_{\min }$ would have been the first mode crossing the horizon during the near-exponential expansion [9]. Thus, for an assumed inflaton potential $V(\phi)$, $k_{\min }$ would have signaled the precise time at which inflation started. As it turns out, however, one could not simultaneously solve the temperature horizon problem and account for the observed fluctuation spectrum in the CMB with this minimum cutoff. If inflation is to work, the sequence of steps preceding (or succeeding) the inflationary phase would necessarily have to be more complicated than has been conjectured thus far.

In this Letter, we take an alternative approach, and simplify the concept of how a scalar field ought to have behaved in order to comply with the observational constraints. Arguably, the most significant, relevant measurements we have to date are (1) the scalar spectral index, $n_{s}=0.9649 \pm 0.0042$, in the power spectrum $P(k)=$ $A_{s}\left(k / k_{0}\right)^{n_{s}-1}$ [3], (2) the amplitude $A_{s}=(2.1 \pm 0.04) \times$ $10^{-9}$ [3] , and now (3) the hard cutoff $k_{\min }=4.34 \pm 0.50 / r_{\mathrm{cmb}}$ [8]. To be clear, none of these measurements argues against the influence of a scalar field, or anisotropies arising from its quantum fluctuations $(\mathrm{QFs})$, but there are now good reasons to question whether its potential is truly inflationary.

Indeed, a significant body of evidence is accumulating in favor of a zero active mass $(\rho+3 p=0)$ equation-of-state in the cosmic fluid, based on the analysis of over 25 different kinds of observation at low and high redshifts. A summary of these results may be found in Table 2 of Ref. [10]. A notable feature of such a universe is that it lacks a horizon problem $[11,12]$, so our failure to build a fully successful inflationary paradigm may simply reflect the fact that the universe does not need it. This is the key assumption we shall make in this Letter. Some additional support for such a cosmological 
model, based on an alternative theoretical concept, may also be found in Ref. [13], with an updated discussion in Ref. [14].

In our step-by-step comparison of the roles played by a non-inflaton $\phi$ versus a conventional inflaton field, we shall distinguish the former from the latter by informally calling it a 'numen' field, representing the earliest manifestation of substance in the universe, with an equation-of-state $\rho_{\phi}+3 p_{\phi}=0$. We shall readily understand why the numen field appears to be superior to an inflaton field in accounting for the measured values of $n_{s}, A_{s}$ and $k_{\min }$. To do so, let us first derive the perturbation growth equation for zero active mass, and see how a numen QF evolves as the universe expands. The essential steps are by now well known, having appeared in both the primary and secondary literature, so we here show only the key results and refer the reader to several other influential publications for the details.

The perturbed Friedmann-Lemaître-Robertson-Walker (FLRW) spacetime for the linearized scalar fluctuations is given by the line element [6,15-17]

$$
\begin{aligned}
d s^{2}= & (1+2 A) d t^{2}-2 a(t)\left(\partial_{i} B\right) d t d x^{i} \\
& -a^{2}(t)\left[(1-2 \psi) \delta_{i j}+2\left(\partial_{i} \partial_{j} E\right)+h_{i j}\right] d x^{i} d x^{j},
\end{aligned}
$$

where indices $i$ and $j$ denote spatial coordinates, $a(t)$ is the expansion factor, and $A, B, \psi$ and $E$ describe the scalar metric perturbations, while $h_{i j}$ are the tensor perturbations.

For small perturbations about the homogeneous numen field $\phi_{0}(t)$,

$\phi(t, \mathbf{x})=\phi_{0}(t)+\delta \phi(t, \mathbf{x})$,

one can identify in the comoving frame the curvature perturbation $\Theta$ on hypersurfaces orthogonal to comoving worldlines [15] as a gauge invariant combination of the metric perturbation $\psi$ and the scalar field perturbation $\delta \phi$ :

$\Theta \equiv \psi+\left(\frac{H}{\dot{\phi}}\right) \delta \phi$

Then, expanding $\Theta$ in Fourier modes, and inserting the linearized metric (Eq. 1) into Einstein's equations, one derives the perturbed equation of motion

$\Theta_{k}^{\prime \prime}+2\left(\frac{z^{\prime}}{z}\right) \Theta_{k}^{\prime}+k^{2} \Theta_{k}=0$,

where overprime denotes a derivative with respect to conformal time $d \tau=d t / a(t)$, and

$z \equiv \frac{a(t)\left(\rho_{\phi}+p_{\phi}\right)^{1 / 2}}{H}$.
Since $\phi_{0}$ is homogeneous, the energy density $\rho_{\phi}$ and pressure $p_{\phi}$ are simply given as

$\rho_{\phi}=\frac{1}{2} \dot{\phi}^{2}+V(\phi)$

and

$p_{\phi}=\frac{1}{2} \dot{\phi}^{2}-V(\phi)$.

The zero active mass condition $\rho_{\phi}+3 p_{\phi}=0$ therefore constrains the potential to have the form $V(\phi)=\dot{\phi}^{2}$, with the explicit solution

$V(\phi)=V_{0} \exp \left\{-\frac{2 \sqrt{4 \pi}}{m_{\mathrm{P}}} \phi\right\}$

The numen field is therefore a special member of the category of minimally coupled fields explored in the 1980's, designed to produce so-called power-law inflation [18-21]. Unlike the other fields, however, the numen field's zero active mass equation-of-state renders it the sole member of this class that actually does not inflate, since $a(t)=t / t_{0}$, in terms of the age of the universe, $t_{0}$. (This normalization of the expansion factor is possible as long as the FLRW metric is spatially flat, which all the observations strongly suggest.)

It is straightforward to see that the conformal time may therefore be written $\tau(t)=t_{0} \ln a(t)$, when the zero of $\tau$ is chosen to coincide with $t=t_{0}$. Thus, $z=m_{\mathrm{P}} a(t) / \sqrt{4 \pi}$, in terms of the Planck mass $m_{\mathrm{P}} \equiv 1 / \sqrt{G}$, so that $z^{\prime} / z=$ $1 / t_{0}$ and $z^{\prime \prime} / z=1 / t_{0}^{2}$. If we now follow the conventional approach of rewriting Eq. (4) using the Mukhanov-Sasaki variable $u_{k} \equiv z \Theta_{k}$, the resulting curvature perturbation equation becomes

$u_{k}^{\prime \prime}+\alpha_{k}^{2} u_{k}=0$

where

$\alpha_{k} \equiv \frac{1}{t_{0}} \sqrt{\left(\frac{2 \pi R_{\mathrm{h}}}{\lambda_{k}}\right)^{2}-1}$,

and $\lambda_{k} \equiv 2 \pi a(t) / k$ is the proper wavelength of mode $k$. The quantity $R_{\mathrm{h}} \equiv c / H=c t$ is the apparent (or gravitational) radius [22] which, as one can see, coincides with the Hubble horizon in a spatially flat universe. The frequency $\alpha_{k}$ is critical to understanding how and why QFs in the numen field are far better suited to the above measurements than those in an inflaton field.

This advantage may be realized first and foremost by considering the explanation provided by Eq. (10) for the origin of $k_{\text {min }}$. The most significant departure of this frequency from its inflaton counterpart is that both $R_{\mathrm{h}}$ and $\lambda_{k}$ scale exactly 
the same way with time. The ratio $R_{\mathrm{h}} / \lambda_{k}$ or, equivalently, $k R_{\mathrm{h}} / a(t)$, is therefore constant for each mode $k$. Numen QFs do not cross back and forth across the horizon, so once the wavelength of a mode is established upon exiting into the semi-classical universe, it remains a fixed fraction of $R_{\mathrm{h}}$ as they expand with time.

The growth Eq. (9) has analytic solutions

$u_{k}(\tau)=\left\{\begin{array}{ll}B(k) e^{ \pm i \alpha_{k} \tau} & \left(2 \pi R_{\mathrm{h}}>\lambda_{k}\right) \\ B(k) e^{ \pm\left|\alpha_{k}\right| \tau} & \left(2 \pi R_{\mathrm{h}}<\lambda_{k}\right)\end{array}\right.$,

showing that all modes $u_{k}$ with a wavelength smaller than $2 \pi R_{\mathrm{h}}$ oscillate, while the super-horizon ones do not, echoing some of the physical characteristics we encounter with a traditional inflaton field. But here the mode with the longest wavelength relevant to the formation of structure is therefore always the one for which $\lambda_{k}(t)=2 \pi R_{\mathrm{h}}(t)$, which happens to be $k_{\min }^{\mathrm{N}}=1 / t_{0}$. (In spite of its appearance, this equality does not constitute a coincidence, because $a(t)$ is itself normalized to 1 at $t_{0}$.)

We shall now show that $k_{\min }$ clearly represents the emergence of the first QF mode out of the Planck regime by interpreting it as a measure of $k_{\min }^{\mathrm{N}}$. Defining the Planck scale as the length $\lambda_{\mathrm{P}}$ for which the Compton wavelength $\lambda_{\mathrm{C}} \equiv 2 \pi / m$ for mass $m$ equals its Schwarzschild radius $R_{\mathrm{h}} \equiv 2 G m$, we find that $\lambda_{\mathrm{P}} \equiv \sqrt{4 \pi G}$. Given that $\lambda_{\mathrm{C}}$ grows as $R_{\mathrm{h}}$ shrinks, quantum mechanics does not actually offer any clear insight into how one should handle modes with wavelengths shorter than $2 \pi \lambda_{\mathrm{P}}$ (the factor $2 \pi$ arising from the definition of $\lambda_{\mathrm{P}}$ in terms of $R_{\mathrm{h}}$ ). This is a serious problem for inflationary cosmology because the fluctuation amplitude $A_{s}$ observed in the $\mathrm{CMB}$ requires $\mathrm{QF}$ modes to have been seeded well before the Planck time $t_{\mathrm{P}}=\lambda_{\mathrm{P}}$, giving rise to what is commonly referred to as the "Trans-Planckian Problem" [23].

But a numen field can avoid this fundamental inconsistency altogether if we argue that each mode $k$ emerges into the semi-classical universe when its wavelength $\lambda_{k}$ equals $2 \pi \lambda_{\mathrm{P}}$, after which it evolves according to the oscillatory solution in Eq. (11). As we shall see, the fact that each succeeding $k$ emerges at later times then naturally produces the near scale-free power spectrum $P(k)$ with $n_{s} \sim 1$. The notion that modes may have been born at a particular spatial scale has already been considered in other guises by several other authors, notably Hollands and Wald [24] though, in their case, the fundamental scale was not related to $\lambda_{\mathrm{P}}$. Other considerations supporting this concept had also been presented in Refs. [25,26].

For the numen field, however, we know that the fundamental scale has to be $\lambda_{\mathrm{P}}$ for the following simplethough quite compelling-reason. From the expression $k=$ $2 \pi a(t) / \lambda_{k}(t)$, we see that the observed value of $k_{\min }$ defines the time $t_{\min }$ at which the very first mode emerged into the semi-classical universe. Thus, $t_{\min }=\frac{4.34 t_{\mathrm{P}}}{\ln \left(1+z_{\mathrm{cmb}}\right)}$

where $z_{\mathrm{cmb}}$ is the redshift at the last scattering surface. In $\Lambda \mathrm{CDM}, z_{\mathrm{cmb}} \sim 1000$, for which $t_{\min } \sim 0.63 t_{\mathrm{p}}$. Of course, we don't know what the exact value of $z_{\mathrm{cmb}}$ will be if we modify the expansion history, but the value of $t_{\min }$ is only weakly dependent on this redshift anyway. For example, even if we were to adopt an extremely different value $z_{\mathrm{cmb}}=50$, the implied first emergence would have occurred at $t_{\min } \sim$ $1.1 t_{\mathrm{P}}$

Thus, the (spatially) largest numen mode measured in the CMB had to emerge from the Planck scale at approximately the Planck time. In other words, the measured value of $k_{\min }$ corresponds to the very first mode that could have physically exited the Planck domain shortly after the Big Bang. This situation is quite unique among cosmological scalar fields, inflaton or otherwise, for here the macroscopic anisotropies in the CMB are shown to be directly coupled to QFs at the Planck scale, an interpretation of $k_{\min }$ that supports the viability of a numen field as an explanation for the origin of structure in the early universe.

As we explore this concept further, we also uncover a natural mechanism for generating a near scale-free power spectrum $P(k)$, that does not rely on the poorly motivated use of a Bunch-Davies vacuum below the Planck scale for the QF normalization $B(k)$ in Eq. (11) [27], where wavelengths shorter than $\lambda_{\mathrm{C}}$ are difficult to interpret quantum mechanically. Nor does this mechanism rely on the uncertain transition of modes back and forth across the horizon, as needs to happen for an inflaton field.

To calculate the QF power spectrum $P(k)$, it is necessary to establish the normalization $B(k)$ of the modes, which is typically done by minimizing the expectation value of the Hamiltonian. But in time-dependent spacetimes, such as inflationary $\Lambda \mathrm{CDM}$, the effects of curvature make the frequencies time dependent. The solution in that case is to seed the fluctuations in the remote past, where the observable modes today presumably had a wavelength much smaller than the horizon. They were therefore unaffected by gravity and could thus be normalized as in Minkowski space, with $B(k)=1 / \sqrt{2 k}$ [17]. This approach defines the BunchDavies vacuum [27] though, as noted earlier, it is subject to a possible trans-Planckian inconsistency.

But this problem completely disappears for the numen field, even though the modes would have emerged at the Planck scale, for the simple reason that the zero active mass condition makes the frame into which the modes emerged from the Planck domain be geodesic. That is, although the Hubble frame was expanding, it was nonetheless in free fall, with zero internal acceleration. This is confirmed quantitatively by the fact that the frequencies $\alpha_{k}$ in Eq. (10) are timeindependent, since both $R_{\mathrm{h}}$ and $\lambda_{k}$ change with time in the 
same way. We shall therefore set $B(k)=1 / \sqrt{2 \alpha_{k}}$ for the numen QFs.

After time $t_{\min }$, which we now understand was effectively $t_{\mathrm{P}}$, modes continued to exit from the Planck domain, but always with a wavelength $\lambda_{k}=2 \pi \lambda_{\mathrm{P}}$. That is, $k=a(t) / \lambda_{\mathrm{P}}$, suggesting that mode $k$ exited at time

$t_{k} \equiv t_{0} \lambda_{\mathrm{P}} k$

Then, from Eq. (11) and the definition of $u_{k}$, we find that

$\left|\Theta_{k}\right|^{2}=\frac{2 \pi}{m_{\mathrm{P}}^{2}} \frac{1}{\alpha_{k} a^{2}}$.

The power spectrum $P_{\Theta}(k)$ is defined according to

$P_{\Theta}(k) \equiv \frac{k^{3}}{2 \pi^{2}}\left|\Theta_{k}\right|^{2}$,

and we therefore see from Eqs. (10), (14) and (15) that

$P_{\Theta}(k)=\frac{1}{(2 \pi)^{2}}\left[\frac{a\left(t_{k}\right)}{a(t)}\right]^{2}\left\{1-\left(\frac{k_{\min }^{\mathrm{N}}}{k}\right)^{2}\right\}^{-1 / 2}$.

What is still missing from this picture is a firm understanding of how classicalization converts homogeneous, isotropic quantum fluctuations into classical anisotropies. This is a common problem with all models invoking a quantum origin for the perturbations, and has not yet been solved [28-33]. It is very likely, however, that the dynamics of classicalization is associated with a particular length (or energy) scale, $L_{*}$, analogous to the Planck scale $\lambda_{\mathrm{P}}[34,35]$, which will here serve the principal purpose of establishing the amplitude of the quantum fluctuations.

Mode $k$ will reach the classicalization scale at $a\left(t_{k}^{*}\right)=$ $L_{*} k / 2 \pi$, corresponding to time $t_{k}^{*}=t_{0} L_{*} k$. And so Eq. (16) may be re-written

$P_{\Theta}(k)=\frac{1}{(2 \pi)^{2}}\left[\frac{\lambda_{\mathrm{P}}}{L_{*}}\right]^{2}\left\{1-\left(\frac{k_{\mathrm{min}}^{\mathrm{N}}}{k}\right)^{2}\right\}^{-1 / 2}$,

which should then be compared directly with the observed power spectrum $P(k)=A_{s}\left(k / k_{0}\right)^{n_{s}-1}$ in the CMB [3]. We find from the measured value of $A_{s}$ that $L_{*} \sim 3.5 \times 10^{3} \lambda_{\mathrm{P}}$. Therefore, with the Planck scale set at $m_{\mathrm{P}} \approx 1.22 \times 10^{19}$ $\mathrm{GeV}$, we infer that classicalization of the numen QFs must have occurred at roughly $3.5 \times 10^{15} \mathrm{GeV}$, remarkably consistent with the energy scale in grand unified theories (GUTs). Unlike our more robust conclusion regarding the origin of $k_{\min }$, this inference may be more speculative, given that the physics describing this process clearly lies beyond the standard model, but it is reasonable to suppose that the numen
QFs oscillated until $t \sim 3.5 \times 10^{3} t_{\mathrm{P}}$, at which point the numen field devolved into GUT particles and the perturbation amplitude remained frozen thereafter.

From Eq. (17), we also see that the numen scalar curvature perturbations have an almost scale-free spectrum, as one can see from the standard definition

$n_{s}=1+\frac{d \ln P_{\Theta}(k)}{d \ln k}=1-\frac{2}{2\left(k / k_{\min }^{\mathrm{N}}\right)^{2}-1}$,

showing that $n_{s}$ is slightly less than 1 , and suggesting that the deviation from a pure scale-free spectrum is due to the difference between $k$ and $\alpha_{k}$ in Eq. (10), which ultimately arises from the Hubble expansion term $\Theta_{k}^{\prime}$ in the growth Eq. (4).

The identification of $k_{\min }$ with the first $\mathrm{QF}$ to have emerged into the semi-classical universe from the Planck domain shortly after the Big Bang is very exciting. At this level of sophistication, we do not yet encounter a trans-Planckian inconsistency though, clearly, probing the physics prior to $t_{\mathrm{P}}$ should be a principal focus of attempts at understanding the nature of $\phi$ and its emergence into the semi-classical universe.

Already, we can say that one of the strongest arguments in favor of the super-horizon freeze-out mechanism during inflation also supports the numen field emergence out of the Planck domain. The coherence of the observed CMB fluctuations [36] requires all of the Fourier modes of a given wavenumber to have an identical phase. With an inflaton field, this happens because they all cross the horizon at the same time. Similarly, all of the numen QFs with a given $k$ have the same phase because they emerge from the Planck domain at a fixed time $t_{k}$. The numen mechanism is a little simpler because it requires fewer steps and, at the same time, appears to avoid at least some of the difficulties faced by conventional inflation.

Our final conclusion from this discussion is that if the early universe was indeed dominated by a single numen field, then we know its potential (Eq. 8) quite precisely. Exponential forms such as these are well motivated in Kaluza-Klein cosmologies, string theories and even supergravity. The measurement of $k_{\min }$ in the CMB fluctuations may therefore catalyze further meaningful development in these areas.

Acknowledgements I am grateful to Amherst College for its support through a John Woodruff Simpson Lectureship.

Data Availability Statement This manuscript has no associated data or the data will not be deposited. [Authors' comment: The only measurements used in this paper are (i) the value of $k_{\min }$, which was previously published in [8]; and (ii) the values of $n_{s}$ and $A_{s}$, which were published in [3].]

Open Access This article is distributed under the terms of the Creative Commons Attribution 4.0 International License (http://creativecomm ons.org/licenses/by/4.0/), which permits unrestricted use, distribution, 
and reproduction in any medium, provided you give appropriate credit to the original author(s) and the source, provide a link to the Creative Commons license, and indicate if changes were made.

Funded by SCOAP ${ }^{3}$.

\section{References}

1. E.L. Wright, C.L. Bennett, K. Gorski, G. Hinshaw, G.F. Smoot, APJ Lett. 464, L21 (1996)

2. C.L. Bennett et al., APJS 148, 97 (2003)

3. Planck Collaboration, A\&A (2018). (Eprint arXiv:1807.06209) (in press)

4. A.H. Guth, Phys. Rev. D 23, 347 (1981)

5. A. Linde, Phys. Lett. B 108, 389 (1982)

6. V.F. Mukhanov, H.A. Feldman, R.H. Brandenberger, Phys. Rep. 215, 203 (1992)

7. C.J. Copi, D. Huterer, D.J. Schwarz, G.D. Starkman, MNRAS 399, 295 (2009)

8. F. Melia, M. López-Corredoira, A\&A 618, A87 (2018)

9. J. Liu and F. Melia, PLB submitted (2019)

10. F. Melia, MNRAS 481, 4855 (2018)

11. F. Melia, A\&A 553 (2013) id. A76

12. F. Melia, EPJ C Lett. 78, 739 (2018)

13. M.V. John \& K.B. Joseph, PRD 61 (2000) id. 087304

14. M.V. John, MNRAS 484, L35 (2019)
15. J.M. Bardeen, PRD 22, 1882 (1980)

16. H. Kodama, M. Sasaki, Prog. Theor. Phys. Suppl. 78, 1 (1984)

17. B.A. Bassett, S. Tsujikawa, D. Wands, Rev. Mod. Phys. 78, 537 (2006)

18. L.F. Abbott, M.B. Wise, Nucl. Phys. 244, 541 (1984)

19. F. Lucchin, S. Materrese, PRD 32, 1316 (1985)

20. J. Barrow, PLB 187, 12 (1987)

21. A.R. Liddle, PLB 220, 502 (1989)

22. F. Melia, Am. J. Phys. 86, 585 (2018)

23. J. Martin, R.H. Brandenberger, PRD 63, 123501 (2001)

24. S. Hollands, R.M. Wald, Gen. Relativ. Gravit. 34, 2043 (2002)

25. R.H. Brandenberger, P.M. Ho, PRD 66, 023517 (2002)

26. S.F. Hassan, M.S. Sloth, Nucl. Phys. B 674, 434 (2003)

27. T.S. Bunch, P.C.W. Davies, Proc. R. Soc. A 360, 117 (1978)

28. R. Penrose, The Road to Reality (Vintage Books, New York, 2004), p. 861

29. A. Perez, H. Sahlmann, D. Sudarsky, CQG 23, 2317 (2006)

30. V.F. Mukhanov, Physical Foundations of Cosmology, vol. 340 (Cambridge University Press, Cambridge, 2005)

31. S. Weinberg, Cosmology (Oxford University Press, Oxford, 2008), p. 447

32. D.H. Lyth, A.R. Liddle, The Primordial Density Perturbation, vol. 386 (Cambridge University Press, Cambridge, 2009)

33. G.R. Bengochea, P. Canate, D. Sudarsky, PLB 743, 484 (2015)

34. N. Brouzakis, J. Rizos, N. Tetradis, PLB 708, 170 (2012)

35. G. Dvali, C. Gomez, JCAP 1207, 015 (2012)

36. S. Dodelson, AIP Conf. Proc. 689, 184 (2003) 